\title{
COLLIDING STRING WAVES AND DUALITY
}

\author{
Ashok Das $^{a}$, J. Maharana $^{b}$ and A. Melikyan ${ }^{a}$ \\ ${ }^{a}$ Department of Physics and Astronomy \\ University of Rochester, NY 14627-0171, USA \\ ${ }^{b}$ Max-Planck-Institut für Gravitationsphysik \\ Albert-Einstein-Institut \\ 14476 Golm, Germany \\ and \\ Institute of Physics, \\ Bhubaneswar - 751005, India
}

\begin{abstract}
The collision of plane waves corresponding to massless states of closed string is considered in $D$-dimensional space-time. The reduced tree level effective action is known to be manifestly $O(d, d)$ invariant, $d$ being the number of transverse spatial dimensions in the collision process. We adopt a coset space reformulation of the effective two dimensional theory and discuss the relation of this process with classical integrable systems in two dimensions in the presence of gravity. We show how it is possible to generate new backgrounds for the scattering process, from known background solutions to the equations of motion, in the coset reformulation. We present explicit calculations for the case of four space-time dimensions as an illustrative example.
\end{abstract}


Recently, there have been attempts to study collision of plane waves in the frame work of string theory [1, 2]. A scenario is envisaged where infinite plane-fronted waves undergo a head on collision. One of the goals of the investigation of this problem is to gain insight into the mechanism for the creation of the Universe in the pre-big bang $(\mathrm{PBB})$ hypothesis [3, 4 . In this scenario, it is proposed that the Universe, in its infancy, is in the weak coupling regime with low curvature, where we can trust the low energy string effective action. In this phase, we can admit the Minkowski vacuum order by order in the perturbative frame work. The Universe, in this early epoch, can be described as a superposition of massless waves, travelling in all directions, whose energies lie below the string scale. These waves collide in the process of propagation, constructively interfere and eventually gravitational collapse takes place, when over-dense regions are formed. It has been argued by Buonanno, Damour and Veneziano [5] that the interior of an adequately large and collapsing region may give birth to a Universe which possesses attributes of our own Universe. In other words, collisions of plane waves could result in nontrivial geometries in the interaction region of the waves, leading to the creation of a Universe as proposed in the PBB scenario. The singularity, arising from the interaction process of the plane waves, finds an interpretation as a cosmological singularity from the perspective of the PBB proposal. In the study of the scattering process, when one solves the underlying equations in the interaction region, the resulting metric assumes Kasner's form. Moreover, the exponents fulfill the requirements of the PBB hypothesis in the space of admissible parameters. Furthermore, it has been argued that initial states of the PBB cosmology could be thought of as the plane waves associated with the massless spectrum of strings [6]. It is important to note that Kasner solutions have attracted considerable attention from various perspectives $[7,8]$.

It is ideal to consider configurations of plane waves of infinite front undergoing collisions and several interesting results have already emerged in recent studies [1, 2]. In the work of FKV [1], the collision process was studied in four $(D=4)$ dimensional space-time for graviton and dilatonic waves. Subsequently, Bozza and Veneziano [2] examined the case of arbitrary $D$-dimensional space-time (with $D-2=d$ transverse dimensions) and incorporated the presence of the antisymmetric tensor background through implementation of the $O(d, d)$ symmetry, since the effective two dimensional tree level action is invariant under this target space symmetry. We mention in passing that collision of plane waves has been studied in detail in general theory of relativity [9]; however, interest, in this, has been revived since there is an interesting connection between this process and the issue of the creation of the Universe in the PBB scenario. Since the problem under consideration is described by a two dimensional effective action, it is natural to expect an intimate connection with integrable systems [10]. Indeed, there has been a considerable amount of work in investigating a class of two dimensional field theories in curved space-time and super-gravity theories to study their integrability properties 11].

It is expected that the techniques adopted in the study of integrable systems will 
unravel other fascinating features of the problem under consideration. In the past, the symmetries of two dimensional string effective action have been investigated in some detail by several authors 112, 13, 14, 15. Moreover, if we adopt the string frame metric in expressing the reduced action, then the manifest $O(d, d)$ symmetry can be exploited to generate new background configurations from known solutions of the equations of motion.

The purpose of this note is two fold. First, we shall argue how the effective action, studied from a different perspective, can be shown to have a direct connection with integrable systems. This is achieved rather elegantly by adopting the coset space reformulation of the action as was derived by Schwarz and one of the authors [16]. Thus the action can be expressed in a current-current form involving the scalar fields (moduli). Our second result is the special case of collision of waves in four space-time dimensions (i.e., transverse dimensions $d=2$ ). In this case, we start with a background where the antisymmetric tensor field is vanishing along transverse directions while the metric and the dilaton are nonzero. We generate a nontrivial $B$-field along the transverse directions by exploiting a property of the coset reformulation, so that the new background configuration can be extracted rather easily. This technique is applicable to the case with arbitrary $d$ transverse dimensions and is a property of the reformulation alluded to above.

The $D$-dimensional tree level string effective action is

$$
S_{D}=\int d^{D} x \sqrt{-\mathcal{G}} e^{-\phi}\left(R_{\mathcal{G}}+(\partial \phi)^{2}-\frac{1}{12} H_{\mu \nu \rho} H^{\mu \nu \rho}\right)
$$

Here $\mathcal{G}_{\mu \nu}$ is the $D$-dimensional metric in the string frame, $\mathcal{G}$ is its determinant and $R_{\mathcal{G}}$ is the curvature scalar derived from this metric. Note that $\phi$ is the dilaton and $H_{\mu \nu \rho}=\partial_{\mu} B_{\nu \rho}+\partial_{\rho} B_{\mu \nu}+\partial_{\nu} B_{\rho \mu}$ is the field strength associated with the anti-symmetric field $B_{\mu \nu}$. When one studies the problem of head on collision of two plane-front waves of infinite extension, it is possible to define a coordinate system where the waves travel along $x^{1}$ direction. If we assume that there is translational symmetry in the transverse directions, then there will be Abelian isometries along these, $D-2=d$, directions. Thus the process is described by a two dimensional reduced effective effective action, which can be derived following the prescription of ref. 16]

$$
S=\int d x^{0} d x^{1} \sqrt{-g} e^{-\bar{\phi}}\left(R+(\partial \bar{\phi})^{2}+\frac{1}{8} \operatorname{Tr}\left(\partial_{\alpha} M^{-1} \partial^{\alpha} M\right)\right)
$$

In general, there will be additional terms in the reduced action corresponding to $d$ Abelian gauge fields from the original metric and another set of $d$ gauge fields from the antisymmetric tensor $B_{\mu \nu}$ as a result of dimensional reduction [16]. Furthermore, there would have been the field strength of the two dimensional tensor field $B_{\alpha \beta}$ where $\alpha, \beta=0,1$ are spacetime indices. Since we are effectively in two space-time dimensions, we have dropped the gauge field terms and, in the same spirit, have not kept field strengths of $B_{\alpha \beta}$, which can be removed if it depends only on coordinates 
$x^{0}$ and $x^{1}$. The other terms appearing in the above action are defined as follows: $g_{\alpha \beta}$ with $\alpha, \beta=0,1$ is the two dimensional space-time metric, and $R$ is the corresponding curvature scalar. The shifted dilaton is $\bar{\phi}=\phi-\frac{1}{2} \log \operatorname{det} G$, with $G$ the metric corresponding to the transverse coordinates $x^{i}, i=2,3 . ., D-1$. $M$ is a $2 d \times 2 d$ symmetric matrix

$$
M=\left(\begin{array}{cc}
G^{-1} & -G^{-1} B \\
B G^{-1} & G-B G^{-1} B
\end{array}\right)
$$

where $B$ represents the moduli coming from the reduction of the $B$-field in $D$ spacetime dimensions. The symmetric nature of $M$ is evident since $G$ is symmetric and $B$ is antisymmetric.

The reduced effective action is invariant under global $O(d, d)$ transformations

$$
\begin{gathered}
g_{\alpha \beta} \rightarrow g_{\alpha \beta}, \quad \bar{\phi} \rightarrow \bar{\phi} \\
M \rightarrow \Omega^{T} M \Omega
\end{gathered}
$$

where $\Omega \in O(d, d)$ is the transformation matrix. The $O(d, d)$ metric

$$
\eta=\left(\begin{array}{ll}
0 & 1 \\
1 & 0
\end{array}\right)
$$

remains invariant under the non-compact global transformations, 1 being the $d$ dimensional unit matrix. Furthermore, it is easy to check that the matrix $M$ belongs to $O(d, d)$.

The relevant field equations are

$$
\begin{gathered}
R+2 g^{\alpha \beta} D_{\alpha} D_{\beta} \bar{\phi}-g^{\alpha \beta} \partial_{\alpha} \bar{\phi} \partial_{\beta} \bar{\phi}+\frac{1}{8} g^{\alpha \beta} \operatorname{Tr}\left(\partial_{\alpha} M^{-1} \partial_{\beta} M\right)=0 \\
R_{\alpha \beta}+D_{\alpha} D_{\beta} \bar{\phi}+\frac{1}{8} \operatorname{Tr}\left(\partial_{\alpha} M^{-1} \partial_{\beta} M\right)=0
\end{gathered}
$$

which result from the variation of the effective action with respect to the shifted dilaton and the metric $g_{\alpha \beta}$ respectively. The equation

$$
D^{\alpha} D_{\alpha} e^{-\bar{\phi}}=0
$$

follows from the above two equations in a straightforward manner. The equation of motion for $M_{i j}$ is

$$
\partial_{\alpha}\left(e^{-\bar{\phi}} \sqrt{-g} g^{\alpha \beta} M^{-1} \partial_{\beta} M\right)=0
$$

which is obtained by a constrained variation of the effective action, taking account of the fact that $M \in O(d, d)$. Equations ([0)-(10) can be simplified further in the light cone coordinates, if we recall that the two dimensional metric can be written as the flat metric times a conformal factor, i.e., $g_{\alpha \beta}=e^{F} \eta_{\alpha \beta}$. In this case, the relevant equations take a rather simple form as has been shown in [2]

$$
\partial_{u} \partial_{v} e^{-\bar{\phi}}=0
$$


which is also equivalent to $\partial_{u} \partial_{v} \bar{\phi}=\partial_{u} \bar{\phi} \partial_{v} \bar{\phi}$ and

$$
\begin{gathered}
\partial_{u}\left(e^{-\bar{\phi}} M^{-1} \partial_{v} M\right)+\partial_{v}\left(e^{-\bar{\phi}} M^{-1} \partial_{u} M\right)=0 \\
\partial_{u}^{2} \bar{\phi}-\partial_{u} F \partial_{u} \bar{\phi}+\frac{1}{8} \operatorname{Tr}\left(\partial_{u} M^{-1} \partial_{u} M\right)=0 \text {, another equation with } u \rightarrow v \\
\partial_{u} \partial_{v} \bar{\phi}-\partial_{u} \partial_{v} F+\frac{1}{8} \operatorname{Tr}\left(\partial_{u} M^{-1} \partial_{v} M\right)=0
\end{gathered}
$$

where $u=\frac{1}{\sqrt{2}}\left(x^{0}-x^{1}\right)$ and $v=\frac{1}{\sqrt{2}}\left(x^{0}+x^{1}\right)$.

Let us recapitulate very briefly how solutions are obtained for the scattering of the plane waves. The waves collide at $u=0$ and $v=0$ which corresponds to $x^{0}=x^{1}=0$. The fronts of the two waves are defined to be at $u=0$ and $v=0$ respectively. In order to describe the collision process, it is convenient to divide space-time into four regions. First, one considers a region which is in front of the plane waves, called region $I$ such that $u, v<0$ and this is before the collision occurs. The metric is Minkowskian here and the string coupling is small, so that we are in the perturbative regime. Here, for the dilaton we have $\phi=\phi_{0}$, with $e^{\phi_{0}}<<1$ and we can fix $\bar{\phi}=0$; and take $B=0$. The line element is

$$
d s_{I}^{2}=-2 d u d v+d x^{i} d x_{i}
$$

in this region and we adopt the convention of summation over repeated indices throughout. Before the collision occurs, a wave is coming from the left, called region $I I$, where $u>0, v<0$ and the backgrounds $G_{i j}^{I I}(u), B_{i j}^{I I}(u)$ and $\phi^{I I}(u)$ are only $u$-dependent and the superscript specifies that they are in region $I I$. The field equations enable us to choose the conformal factor, $F=0$ in region $I I$ and the line element has the following form

$$
d s_{I I}^{2}=-2 d u d v+G_{i j}^{I I}(u) d x^{i} d x^{j}
$$

The other wave front is coming from the right, i.e., $v>0$ and $u<0$, which is denoted by $I I I$. Here the backgrounds depend only on $v$ and they are designated with the superscript $I I I$. We shall not display the line element explicitly; it has the same form as that for $I I$, except that $G_{i j}^{I I}(u)$ is replaced by $G_{i j}^{I I}(v)$. Finally, we have region $I V$, where interaction takes place and the conformal factor, $F(u, v)$, makes its appearance in equations and the backgrounds $G_{i j}^{I V}, B_{i j}^{I V}$ and $\phi^{I V}$ depend on both $u$ and $v$ so that the line element takes the form

$$
d s_{I V}^{2}=-2 e^{F(u, v)} d u d v+G_{i j}^{I V}(u, v) d x^{i} d x^{j}
$$

The solutions to background equations of motion for this system in the string theory have been discussed in [1, 2], especially the solution for the diagonal form of $G$ has been dealt with in detail. Our goal, in the first step, is to show the connection between 
this problem and integrable systems [10]. In order to achieve this, first we have to cast the action in a different form.

We may recall that the reduced effective action admits a coset space reformulation as was demonstrated in ref. [16]. One introduces a triangular matrix form for $M$, such that $M=V V^{T}$ and

$$
V=\left(\begin{array}{cc}
E^{-1} & 0 \\
B E^{-1} & E^{T}
\end{array}\right)
$$

where $B$ is the antisymmetric tensor $B_{i j}$ and $E$ is a vielbein such that $\left(E^{T} E\right)_{i j}=G_{i j}$. Also it is easy to show that $V, V^{T} \in O(d, d)$. It was shown that one can introduce an arbitrary matrix $V$ which belongs to $O(d, d)$ (not the special form given above) and write an action which is invariant under global $O(d, d)$ transformation as well as under local gauge transformations of its maximal compact subgroup $O(d) \times O(d)$. Then, one can make a gauge choice to obtain the form of the reduced action that appears in standard dimensional reduction with manifest $O(d, d)$ symmetry. Of course, for the problem at hand, we shall adopt the results of [16] accordingly. First, we introduce a transformation to go over to the diagonal form of $O(d, d)$ metric, $\sigma=\left(\begin{array}{cc}\mathbf{1} & 0 \\ 0 & -\mathbf{1}\end{array}\right), \mathbf{1}$ being the $d \times d$ unit matrix, through the relation

$$
L^{T} \eta L=\sigma, \quad L=\frac{1}{\sqrt{2}}\left(\begin{array}{cc}
\mathbf{1} & -\mathbf{1} \\
\mathbf{1} & \mathbf{1}
\end{array}\right)
$$

The $M$ and the $V$ matrices transform as follows:

$$
M \rightarrow \tilde{M}=L^{T} M L, \quad V \rightarrow \tilde{V}=L^{T} V L
$$

Let us next define a current

$$
\tilde{V}^{-1} \partial_{\alpha} \tilde{V}=P_{\alpha}+Q_{\alpha}
$$

where $\tilde{V}^{-1} \partial_{\alpha} \tilde{V} \in O(d, d)$ and $Q_{\alpha} \in O(d) \times O(d)$, the maximal compact subgroup. It is straightforward to show that

$$
\operatorname{Tr}\left(\partial_{\alpha} M^{-1} \partial_{\beta} M\right)=-4 \operatorname{Tr} P_{\alpha} P_{\beta}
$$

It follows from the symmetric space automorphism property of the coset $\frac{O(d, d)}{O(d) \times O(d)}$ that $P_{\alpha}^{T}=P_{\alpha}$, which belongs to the complement and $Q_{\alpha}^{T}=-Q_{\alpha}$ as $Q_{\alpha} \in O(d) \times$ $O(d)$. Thus, we can write $P_{\alpha}=\frac{1}{2}\left[\tilde{V}^{-1} \partial_{\alpha} \tilde{V}+\left(\tilde{V}^{-1} \partial_{\alpha} \tilde{V}\right)^{T}\right]$ and then (22) follows in a straightforward manner. We mention another important point in passing, namely, that, under an $O(d) \times O(d)$ gauge transformation $\tilde{V} \rightarrow \tilde{V} h(x), h(x) \in O(d) \times O(d)$, $P_{\alpha}$ and $Q_{\alpha}$ transform as follows

$$
P_{\alpha} \rightarrow h(x)^{-1} P_{\alpha} h(x), \quad Q_{\alpha} \rightarrow h^{-1}(x) Q_{\alpha} h(x)+h^{-1} \partial_{\alpha} h(x)
$$

and this transformation leaves (22) invariant. Notice the form of (22): in flat spacetime, this describes a $\sigma$-model in two dimensions. Furthermore, it is possible to 
introduce a constant spectral parameter and a zero curvature condition by taking a suitable combination of $P_{\alpha}$ and $Q_{\alpha}$ to show the integrability properties of the classical theory following well known procedures [10]. For the case at hand, the full action consists of kinetic energy terms for the conformal factor $F$ and the shifted dilaton $\bar{\phi}$ in addition to the piece given by (22). One can scale the metric $g_{\alpha \beta} \rightarrow e^{\bar{\phi}} g_{\alpha \beta}$ to get rid of the kinetic term of $\bar{\phi}$ in action (2). Then, for the modified action, one can follow the procedure described in [1] to establish relation of this process with classical integrable systems. Let us consider the special case, as an illustrative example, when the metric $G_{i j}$ is diagonal as was the case in [1, 2] and $B_{i j}=0$. The corresponding $V$-matrix is symmetric and so is $\tilde{V}$. Thus it follows from (21) that $Q_{\alpha}=0$ and one needs to suitably reformulate integrability conditions for this case. When one studies integrability properties of two dimensional models in curved space-time, the spectral parameter assumes space-time dependence. Furthermore, $\tilde{V}\left(x^{0}, x^{1}\right) \rightarrow \hat{V}\left(x^{0}, x^{1}, t\right)$ such that

$$
\begin{aligned}
\hat{V}^{-1} \partial_{v} \hat{V} & =\frac{1-t}{1+t} P_{v} \\
\hat{V}^{-1} \partial_{u} \hat{V} & =\frac{1+t}{1-t} P_{u}
\end{aligned}
$$

The equations of motion and integrability condition

$$
\partial_{\alpha}\left(\hat{V}^{-1} \partial_{\beta} \hat{V}\right)-\partial_{\beta}\left(\hat{V}^{-1} \partial_{\alpha} \hat{V}\right)+\left[\hat{V}^{-1} \partial_{\alpha} \hat{V}, \hat{V}^{-1} \partial_{\beta} \hat{V}\right]=0
$$

are compatible provided the spectral parameter satisfies

$$
\partial_{\alpha} t=-\frac{1}{2} \epsilon_{\alpha \beta} \partial^{\beta}\left(e^{-\bar{\phi}}\left(t+\frac{1}{t}\right)\right)
$$

The solution to the above first order equation is

$$
\frac{1-t}{1+t}=\sqrt{\frac{w-e^{-\bar{\phi}(u)}}{w+e^{-\bar{\phi}(v)}}}
$$

where $w$ is an integration constant. We shall see that, in region $I V, e^{-\bar{\phi}}$ is expressed as a sum of two solutions, one depending on $u$ and the other on $v$. The next step is to solve for the monodromy matrix. It is well known that the linear system, defined through (24), is invariant under the generalization of symmetric space automorphism. One defines

$$
\tau^{\infty} \hat{V}(t)=\left(\hat{V}^{T}\right)^{-1}\left(\frac{1}{t}\right)
$$

In terms of Lie algebra elements, this implies $P_{\alpha} \rightarrow-P_{\alpha}, \quad t \rightarrow \frac{1}{t}$. Furthermore, the current $\hat{V}^{-1} \partial_{\alpha} \hat{V}$ is invariant under $\tau^{\infty}$ transformation. The monodromy matrix

$$
\mathcal{M}=\hat{V} \tau^{\infty}(\hat{V})^{-1}=\hat{V}(x, t) \hat{V}^{T}\left(x, \frac{1}{t}\right)
$$


plays a fundamental role in the reconstruction of solutions as well as in the study of integrability properties from this point of view. We shall present the explicit form of $\mathcal{M}$ for the four dimensional case, when we discuss solutions.

Another aspect of this reformulation is that one can generate new background configurations from known solutions as follows: First solve for equations of motion for a given background. Then implement an $O(d, d)$ transformation on $\tilde{V}$. In general, the resulting $V$ obtained from transformed $\tilde{V}$ will not maintain its triangular form, which is quite essential to get the desired form of the $M$-matrix. At this stage, one can further make a transformation $h \in O(d) \times O(d)$ to bring the transformed $V$, call it $V^{\prime}$, to a triangular form.

Let us consider, to be specific, the scattering of plane fronted waves in $D=4$ for which the duality symmetry is $O(2,2)$. Thus $G_{i j}$ and $B_{i j}$ are $2 \times 2$ matrices. If we choose a background configuration where $G_{i j}$ is diagonal and $B_{i j}=0$, then

$$
V=\left(\begin{array}{cc}
E^{-1} & 0 \\
0 & E^{T}
\end{array}\right)
$$

Here, $E$ is the corresponding zweibein with a choice $E=\left(\begin{array}{cc}E_{1} & 0 \\ 0 & E_{2}\end{array}\right)$ and that $G=$ $E^{T} E$. We recall that under a global $O(2,2)$ transformation, $V \rightarrow \Omega^{T} V$ with $\Omega \in$ $O(2,2)$. Let us choose

$$
\Omega=\left(\begin{array}{cc}
a \mathbf{1} & b \epsilon \\
-c \epsilon & d \mathbf{1}
\end{array}\right)
$$

where 1 is unit $2 \times 2$ matrix and $\epsilon$ is the antisymmetric $2 \times 2$ matrix. Note that $a, b, c$ and $d$ are real numbers satisfying $a d-b c=1$, in order that the $4 \times 4$ matrix $\Omega \in O(2,2)$. Under such a transformation,

$$
V \rightarrow \Omega^{T} V=\left(\begin{array}{cc}
a E^{-1} & c \epsilon E^{T} \\
-b \epsilon E^{-1} & d E^{T}
\end{array}\right)
$$

Thus, as mentioned, the transformed $V$ is not in the desired triangular form, although we can read off the new transformed backgrounds to be

$$
E^{\prime}=\frac{E}{a}, \quad B^{\prime}=-\frac{b}{c} \epsilon
$$

Note that the resulting $B^{\prime}$ is just a constant.

However, let us recall that we also have the freedom of making a local $O(2) \times O(2)$ transformation under which, $\tilde{V} \rightarrow \tilde{V} h(x), h(x) \in O(2) \times O(2)$. Let us choose the $4 \times 4$ matrix $h$ to be space-time independent and of the form

$$
h=\left(\begin{array}{ll}
\gamma \mathbf{1} & \delta \epsilon \\
\delta \epsilon & \gamma \mathbf{1}
\end{array}\right)
$$

It is easy to check that, $h h^{T}=h^{T} h=\mathbf{1}$ provided, $\gamma^{2}+\delta^{2}=1$. The $O(2) \times O(2)$ nature of the matrix becomes more transparent, if we go to the diagonal basis for the 
metric of $O(2,2)$ where we see that $h \rightarrow L^{T} h L=\operatorname{diag}(\gamma \mathbf{1}+\delta \epsilon, \gamma \mathbf{1}-\delta \epsilon)$ and the off diagonal elements are zero. After $V$ gets transformed under both $\Omega$ and $h$, we finally get

$$
V \rightarrow \Omega^{T} V h=\left(\begin{array}{cc}
a \gamma E^{-1}+c \delta E^{T} \epsilon & a \delta E^{-1} \epsilon+c \gamma \epsilon E^{T} \\
-b \gamma \epsilon E^{-1}+d \delta E^{T} \epsilon & -b \delta \epsilon E^{-1} \epsilon+d \gamma E^{T}
\end{array}\right)
$$

If this transformed $V$ is to be of triangular form, then its " 12 " component is to be set to zero, $a \delta E^{-1} \epsilon+c \gamma \epsilon E^{T}=0$ which determines, $\gamma$ and $\delta$ to be

$$
\gamma=\frac{a}{\sqrt{a^{2}+c^{2} E_{1}^{2} E_{2}^{2}}}, \quad \delta=-\frac{c E_{1} E_{2}}{\sqrt{a^{2}+c^{2} E_{1}^{2} E_{2}^{2}}}
$$

and they automatically satisfy the requirement $\gamma^{2}+\delta^{2}=1$. The new backgrounds, denoted by primes (not to be confused with derivatives) are

$$
\begin{gathered}
G^{\prime}=\left(a^{2} G^{-1}-c^{2} \epsilon G \epsilon\right)^{-1} \\
B^{\prime}=-\frac{a b+c d E_{1}^{2} E_{2}^{2}}{a^{2}+c^{2} E_{1}^{2} E_{2}^{2}} \epsilon
\end{gathered}
$$

and we note that the new moduli $B_{i j}^{\prime}$ are space-time dependent. Furthermore, $G_{i j}^{\prime}$ depends on $G$ and $G^{-1}$ and is diagonal. For this choice of $\Omega$ and $h$ we have generated nontrivial backgrounds.

Let us now discuss some of the essential properties of the solutions in region $I V$, since this is the region where collision occurs. When we implement an $O(d, d)$ transformation, the shifted dilaton $\bar{\phi}$ remains invariant. The solutions to the equation of motion, for diagonal metric and $B=0$, has been discussed in [1, 2], where they appear in form of integrals. These results are generalization of the case of pure gravity considered by Szekeres [17] and subsequently by Yurtsever [18. The asymptotic behavior of the solutions are of interest, since one of the aims is to examine whether a singularity appears in the backgrounds. Let us express [1] the diagonal elements $G_{i j}, i, j=2,3$, corresponding to directions $x^{2}$ and $x^{3}$, in the following form $G_{22}=$ $\exp (\lambda+\psi)$ and $G_{33}=\exp (\lambda-\psi)$. Thus $\lambda=\frac{1}{2} \log \operatorname{det} G$. The dilaton, in region $I V$, can be written as

$$
e^{-\bar{\phi}(u, v)}=e^{-\bar{\phi}_{I I}(u)}+e^{-\bar{\phi}_{I I I}(v)}-1
$$

That it is a sum of a function of $u$ and of $v$ in region $I V$, follows from the equation of motion for $\bar{\phi}$ as well as the fact that the solutions should match smoothly across the boundaries of the different regions. It is convenient to introduce a new set of variables

$$
r=2 \exp \left(-\bar{\phi}_{I I I}(v)\right)-1, \quad s=2 \exp \left(-\bar{\phi}_{I I}(u)\right)-1
$$

Thus $r$ and $s$ are functions of the variables $v$ and $u$ respectively. Let us define a set of coordinates

$$
\xi=\frac{(r+s)}{2}=\exp (-\bar{\phi}(u, v)), \quad z=\frac{(r-s)}{2}=e^{-\bar{\phi}_{I I}(u)}-e^{-\bar{\phi}_{I I I}(v)}
$$


Obviously, $\bar{\phi}(u, v)$ is defined only in region $I V$. From now on, we are going to concentrate on the backgrounds only in region $I V$ and therefore, we suppress the subscript $I V$ from all of them. Under the change of variables, the conformal conformal factor appearing in the space-time metric gets affected. The line element in region $I V$ takes the form (subscript not displayed)

$$
d s^{2}=-e^{f} d \xi^{2}+e^{f} d z^{2}+G_{22}\left(d x^{2}\right)^{2}+G_{33}\left(d x^{3}\right)^{2}
$$

where $f=f(r, s)$ is the conformal factor and

$$
\bar{\phi}=-\frac{1}{2} \log (r+s)=-\log \xi
$$

The asymptotic behavior of the functions $\lambda, \psi$, appearing in $G_{i j}$, the conformal factor, $f$ and dilaton $\phi$ are given by

$$
\begin{gathered}
\lambda \sim \kappa(z) \log \xi, \quad \psi \sim A(z) \log \xi \\
f \sim B(z) \log \xi, \quad \phi \sim \kappa(z) \log \xi-1
\end{gathered}
$$

where

$$
\begin{aligned}
\kappa(z)= & \frac{1}{\pi \sqrt{1+z}} \int_{z}^{1} d s\left[(1+s)^{\frac{1}{2}} \lambda(1, s)\right],_{s}\left(\frac{s+1}{s-z}\right)^{\frac{1}{2}} \\
& +\frac{1}{\pi \sqrt{1-z}} \int_{-z}^{1} d r\left[(1+r)^{\frac{1}{2}} \lambda(r, 1)\right],_{r}\left(\frac{r+1}{r+z}\right)^{\frac{1}{2}} \\
A(z)= & \frac{1}{\pi \sqrt{1+z}} \int_{z}^{1} d s\left[(1+s)^{\frac{1}{2}} \psi(1, s)\right],{ }_{s}\left(\frac{s+1}{s-z}\right)^{\frac{1}{2}} \\
& +\frac{1}{\pi \sqrt{1-z}} \int_{-z}^{1} d r\left[(1+r)^{\frac{1}{2}} \psi(r, 1)\right]_{r}\left(\frac{r+1}{r+z}\right)^{\frac{1}{2}} \\
& B(z)=\frac{1}{2}\left(A(z)^{2}+\kappa(z)^{2}\right)-1
\end{aligned}
$$

We can now read off the line element in the asymptotic limit of the metric

$$
d s^{2}=-\xi^{B(z)} d \xi^{2}+\xi^{B(z)} d z^{2}+\xi^{\kappa(z)+A(z)}\left(d x^{2}\right)^{2}+\xi^{\kappa(z)-A(z)}\left(d x^{3}\right)^{2}
$$

If we define the cosmic time $t=\xi^{\frac{(B(z)+2)}{2}}$, then the Kasner exponents can be read off directly to be [1, 2]

$$
p_{1}=\frac{B}{B+2}, \quad p_{2}=\frac{\kappa+A}{B+2}, \quad p_{3}=\frac{\kappa-A}{B+2}
$$

The exponents satisfy the constraints,

$$
p_{1}^{2}+p_{2}^{2}+p_{3}^{2}=1, \quad \phi=\left(p_{1}+p_{2}+p_{3}-1\right) \log \xi
$$


There is a region in the parameter space such that all the exponents are allowed to take negativing values and at the same time they can satisfy the constraints as has been discussed for the four dimensional case [1]. For such a case, there is a curvature singularity, and this singularity has the characteristics of a cosmological singularity, which is very interesting in the PBB scenario.

Now we would like to examine, the singularity structure for the new backgrounds generated through specific $O(d, d)$ transformations introduced earlier. Note that the shifted dilaton, $\bar{\phi}$ remains invariant under $O(d, d)$ transformation. The two elements of the vielbein, $E$ are given by

$$
E_{1}=\exp \left[\frac{1}{2}(\lambda+\psi)\right]=\xi^{\frac{1}{2}(\kappa+A)}, \quad E_{2}=\exp \left[\frac{1}{2}(\lambda-\psi)\right]=\xi^{\frac{1}{2}(\kappa-A)}
$$

Now the transformed metric becomes

$$
G_{22}^{\prime}=\frac{E_{1}}{\left(a^{2}+c^{2} E_{1} E_{2}\right)}, \quad G_{33}^{\prime}=\frac{E_{2}}{\left(a^{2}+c^{2} E_{1} E_{2}\right)}
$$

The explicit forms of the elements, $E_{1}$ and $E_{2}$ are given above. Thus, near the singularity, when $E_{1}, E_{2}$ go to large values, the elements of the transformed metric remain finite. Similarly when $E_{1}, E_{2}$ tend to zero, the structure of $\tilde{G}_{i j}$ tells us that its matrix elements also tend to zero as $E_{1}$ and $E_{2}$ respectively. Of course, the diagonal structure is maintained. Now turning to the dilaton in the transformed theory,

$$
\phi^{\prime}=\phi-\frac{1}{2} \log \left(\frac{\operatorname{det} G}{\operatorname{det} G^{\prime}}\right)=\phi-\log \left(a^{2}+c^{2} e^{\lambda}\right)
$$

Note, however, that in the two extreme limits of $E_{1}, E_{2}$ either tending to zero or to infinity, $B_{i j}$ tends to a constant as is evident from (38).

Let us proceed to derive the monodromy matrix for the above $D=4$ case which in turn will provide connections with integrable system. The relation (24) suggests that $\hat{V}$ is a $4 \times 4$ matrix for our case. Moreover, the requirement of factorization of the monodromy matrix, alluded to earlier, implies that $\hat{V}(t)$ will have a simple pole structure in the spectral parameter $t$. In turn, it ensures that $\mathcal{M}(w)$ also has only simple poles. Now one can suitably modify the steps indicated in [1] to construct the $\hat{V}$ matrix and eventually the monodromy matrix, which has the form

$$
\mathcal{M}(w)=\left(\begin{array}{cccc}
\frac{w_{1}-w}{w_{1}+w} & 0 & 0 & 0 \\
0 & \frac{w_{3}-w}{w_{3}+w} & 0 & 0 \\
0 & 0 & \frac{w_{1}+w}{w_{1}-w} & 0 \\
0 & 0 & 0 & \frac{w_{3}+w}{w_{3}-w}
\end{array}\right)
$$

This form of the monodromy matrix satisfies all the expected properties. Note from (39) that the shifted dilaton, in region $I V$, can be written as sum of two functions: one depends on $u$ and other on $v$, which is similar to the decomposition employed in 
11]. As a consistency check, we have considered the case when $\psi=0$, that appears in metric $G_{i j}$ and one could recover the known results as discussed in 11. Note that, our discussion can be generalized to the case when $G$ is a $d \times d$ matrix as was considered in [2]; however, the number of poles for the $2 d \times 2 d$ dimensional $\hat{V}$ matrix will be more.

To summarize, in this note, we have presented a coset space reformulation of the string effective action to study the collision of plane-fronted waves corresponding to massless states of closed string. In this process, we have expressed the action in a form which makes it suitable to study the integrability properties of the theory. We have shown that the techniques employed in the study of integrable systems in the presence of gravity can be generalized to the case of plane wave scatterings in string theory. The monodromy matrix is derived explicitly for the $D=4$ case. We have presented an illustrative example, in the case of four space-time dimensions, of how one can generate new background configurations from known solutions in the collision process. We have exploited the properties of the coset space reformulation to generate a space-time dependent $B$-field keeping the transformed metric diagonal. We have only used a special type of $O(2,2)$ and $O(2) \times O(2)$ transformation to generate new backgrounds. One can employ more general form, such as space-time dependent $O(2) \times O(2)$ function $h(x)$, for generating a wide variety of background configurations. The approach presented here can be applied to more general situations while considering plane wave scatterings in the context of string theory.

Acknowledgement: One of us (JM) would like to thank G. Veneziano for discussions on their work [2]. We would like to thank H. Nicolai for discussions and suggestions. One of us (JM) acknowledges gracious hospitality of H. Nicolai and the Albert Einstein Institute. This work is supported in part by US DOE Grant No. DE-FG 02-91ER40685. 


\section{References}

1. A. Feinstein, K. E. Kunze and M. A. Vázquez-Mozo, Class. Quant. Grav. 17 (2000) 3599, hep-th/0002070.

2. V. Bozza and G. Veneziano, JHEP 0010 (2000) 035, hep-th/0007159.

3. G. Veneziano, Phys. Lett. B265 (1991) 287; M. Gasperini and G. Veneziano, Astropart. Phys. 1 (1993) 317.

4. J. E. Lidsey, D. Wands and E. J. Copeland, Phys. Rep. C 337 (2000) 343; G. Veneziano, hep-th/0002094 and see http://www.to.infn.it/ gasperin for updated literature in the subject.

5. A. Buonano, T. Damour and G. Veneziano, Nucl. Phys. 543 (1999) 275, hepth/9806230.

6. D. Clancy, J. E. Lidsey and R. Tavakol, Phys. Rev. D58 (1998) 044017; Phys. Rev. D59 (1999) 063511, Phys. Rev. D60 (1999) 043593; Phys. Lett. B451 (1999) 303 and K. E. Kunze, Class. Quant. Grav. 16 (1999) 3795.

7. T. Damour and M. Henneaux, Phys. Rev. Lett. 85 (2000) 920, hep-th/0003139; T. Damour and M. Henneaux, Phys. Lett. B488 (2000) 108, hep-th/0006171.

8. T. Damour, M. Henneaux, B. Julia and H. Nicolai, Phys. Lett. B509 (2001) 323.

9. For discussion on colliding waves see, J. B. Griffiths, Colliding Plane Waves in General Relativity, Oxford University Press, Oxford, UK (1991).

10. A. Das, "Integrable Models", World Scientific, Singapore (1989).

11. There is a vast literature on the subject; we found following two articles very useful which also contain extensive references. H. Nicolai, "Recent Aspects of Quantum Fields", Schladming Lectures, eds. H. Mitter and H. Gausterer, Springer Verlag, Berlin (1991). H. Nicolai, D. Korotkin and H. Samtleben, "Integrable Classical and Quantum Gravity", NATO Advanced Study Institute on Quantum Fields and Quantum Spacetime, Cargese, 1996, hep-th/9612065.

12. J. Maharana, Phys. Rev. Lett. 75 (1995) 205, hep-th/9502001; Mod. Phys. Lett. A11 (1996), hep-th/9502002.

13. J. H. Schwarz, Nucl. Phys. B447 (1995) 137, hep-th/9503078; "Classical Duality Symmetries in Two Dimensions", STRINGS 95, hep-th/9505170; Nucl. Phys. B454 (1995) 427.

14. A. Sen, Nucl. Phys. B447 (1995) 62. 
15. I. Bakas, Nucl. Phys. 428 (1994) 374; S. Mizoguchi, Nucl. Phys. B461 (1996) 155; E. Abdalla and M. C. B. Abdalla. Phys. Lett. B365 (1996) 41; A. K. Biswas, A. Kumar and K. Ray, Nucl. Phys. B453 (1995) 181; A. A. Kehagias, Phys. Lett. B360 (1995) 19.

16. J. Maharana and J. H. Schwarz, Nucl. Phys. B390 (1993) 3, hep-th/9207016.

17. P. Szekeres, J. Math. Phys. 13 (1972) 286.

18. U. Yurtsever, Phys. Rev. D37 (1988) 2803. 